\title{
EFECTO DEL CONTENIDO PROTEICO Y ENERGÉTICO DE DIETAS EN EL CRECIMIENTO DE ALEVINOS DE GAMITANA (Colosssoma macropomum)
}

\author{
Palmira Padilla Pérez ${ }^{1}$
}

\section{RESUMEN}

Se utilizaron alevinos de gamitana, Colossoma macropomum, con peso promedio de $8,13 \mathrm{~g}$, obtenidos por reproducción artificial, colocados en dos estanques de 2640 $\mathrm{m}^{2}$ y $2940 \mathrm{~m}^{2}$ a una densidad de $1 \mathrm{pez} / \mathrm{m}^{2}$, a fin de estudiar el efecto de dos niveles de proteína bruta $(18,50 \%$ y $24,69 \%)$ y de energía bruta $(345,91$ y $353,78 \mathrm{kcal} / \mathrm{g})$ sobre el crecimiento de los peces.

El experimento tuvo una duración de 180 días, durante los cuales los peces fueron alimentados al 3\% de la biomasa total de cada estanque.

Los pesos promedios finales de los peces fueron de 409,97 y 673,20 g, y la biomasa de 1205,31 y $1777,29 \mathrm{~g}$, respectivamente.

La conversión alimenticia aparente (CAA) de los peces fue de 2,7 y 2,9.

Palabras claves: Piscicultura, Colossoma macropomum, proteínas, energía, alimentación.

\begin{abstract}
In the present study gamitana Colossoma macropomum fingerling, were used with an average weight of $8,93 \mathrm{~g}$. they were obtained through artificial reproduction, then placed into two ponds of $2640 \mathrm{~m}^{2}$ and $2940 \mathrm{~m}^{2}$ at a density of 1 fish per $\mathrm{m} 2$. The purpose was to study the effect of two different levels of crude protein $(18,5 \%$ and $24,69 \%)$ and 1 total energy $(342,91$ and $353,78 \mathrm{kcal} / \mathrm{g})$ on fish growth.

1 Instituto de Investigaciones de la Amazonía Peruana-IIAP. Programa de Ecosistemas Acuáticos. Centro Regional de Investigaciones de Loreto. Apartado Postal 784. Telefax 265527. Teléfono 265515/265516. Iquitos-Perú.
\end{abstract}


The experiment had a duration of 180 days, during which the fish were fed $3 \%$ of total biomass of each pond.

Final average weights of the fish were 409,97 and 673,20 g, with a biomass of 1 $205,31 \mathrm{~g}$ and $1777,29 \mathrm{~g}$ respectively.

Feed conversion (FC) of the fish were 2,1:1 and 2,9:1.

\section{INTRODUCCIÓN}

Con el aumento de la demanda de alimento, la necesidad de buscar nuevas alternativas nos llevan a crear tecnologías de cultivo de muchos peces nativos, entre ellos Colossoma macropomum, que ha demostrado grandes ventajas para su cultivo en ambientes artificiales, pudiéndose constituir en una fuente importante en la producción de proteína animal (Luna, 1987).

Pocos son los trabajos que muestran los requerimientos nutricionales del Colossoma macropomum, (Macedo et al., 1981; Saldaña \& López, 1988; Luna, 1987). La mayor parte de los estudios sobre nutrición de peces tropicales se basa en la calidad de los alimentos y no en los requirimientos nutricionales, (Saint-Paul \& Werder, 1977; Macedo, 1979; Luna, 1987).

El Colossoma macropomum es una especie básicamente omnívora y puede aceptar bajos niveles de proteína bruta en su dieta (menos de 25\%), (Saint-Paul \& Werder, 1981). Se asume que sus requerimientos de carbohidratos sean más elevados para aprovechar mejor las proteínas.

Basados en la problemática encima descrita, éste trabajo tiene como principal objetivo evaluar la influencia de dos niveles proteícos y energéticos en el crecimiento de la gamitana.

\section{MATERIALES Y MÉTODOS}

El experimento se realizó en dos estanques de tierra con espejos de agua de 2640 y $2940 \mathrm{~m}^{2}$ respectivamente, pertenecientes al Centro Regional de Investigación CRI-Loreto; Iquitos - Perú.

Se utilizaron alevinos de gamitana, Colossoma macropomum, obtenidos por reproducción artificial. 
Fueron formuladas dos dietas con $18,50 \%$ y $24,69 \%$ de proteína bruta, con los siguientes ingredientes: Harina de pescado, harina de maíz, molluelo de trigo, gallina$\mathrm{za}$, harina de soya, sal y minevit, (Tablas 1 y 2). Los peces fueron alimentados cinco veces por semana a razón de $3 \%$ de la biomasa de cada estanque. La densidad de siembra fue de $1 \mathrm{pez} / \mathrm{m}^{2}$.

Las biometrías se realizaron al inicio del experimento y se repetieron cada 15 días durante los 180 días de duración del estudio, donde se tomaba al azar el 15\% del total de la población, midiéndose la longitud total $(\mathrm{cm})$ y el peso $(\mathrm{g})$.

La temperatura se registró diariamente, mientras que los tenores de oxígeno y conductividad eléctrica del agua se midieron una vez por semana.

El análisis cuantitativo de los datos del crecimiento de los peces se basó en el uso del método deductivo sobre la curva de crecimiento en peso, usando la metodología de Santos (1978). Los promedios obtenidos se utilizaron para aplicar la transformación de Ford-Walford (1946), luego fue aplicado el modelo de Von Bertalanffy (1938):

$$
\mathrm{L}_{T}=\mathbf{L} ¥\left(1-\mathbf{e}^{-\mathbf{k}(\mathrm{T}+\mathrm{To})}\right)
$$

Donde : $\mathrm{L}_{\mathrm{T}}=$ Longitud media en el tiempo

$\mathrm{L} ¥=$ Longitud media asintótica

$\mathrm{e}=$ Base de los logarismos neperianos

$\mathrm{K}=$ Constante (mide la velocidad del crecimiento de peces)

La expresión fue ajustada por el método de los mínimos cuadrados. Para la relación longitud/peso se usó la siguiente expresión:

$$
\mathrm{W}_{\mathbf{T}}=\mathbf{f} \mathbf{L}_{\mathrm{T}}^{\mathrm{q}}
$$

Donde: $\quad \mathrm{W}_{\mathrm{T}} \mathrm{y} \mathrm{L}_{\mathrm{T}}=$ Peso y longitud medidas en el tiempo

$\mathrm{f}=$ Constante, también llamada factor de condición

$\mathrm{q}=$ Constante, siendo \pm 3

La conversión alimenticia aparente (CAA) se calculó según Rangel (1987). Con la ecuación:

$$
\mathrm{CAA}=\frac{\mathrm{GP}}{\mathrm{RC}}=\frac{\text { Peso promedio final }- \text { Peso promedio inicial }}{\text { Cantidad de ración proporcionada }}
$$


Tabla 1. Composición porcentual de las dietas experimentales.

\begin{tabular}{|l|r|r|}
\hline INGREDIENTES & D1 & D2 \\
\hline Harina de pescado & 10,00 & 30,00 \\
\hline Harina de soya & 5,00 & 0,00 \\
\hline Harina de maíz & 40,00 & 44,00 \\
\hline Molluelo de trigo & 35,00 & 15,00 \\
\hline Gallinaza & 9,20 & 10,00 \\
\hline Sal & 0,60 & 0,80 \\
\hline Minevit & 0,20 & 0,20 \\
\hline
\end{tabular}

Tabla 2. Composición bromatológica de las dietas.

\begin{tabular}{|l|r|c|}
\hline \multicolumn{1}{|c|}{ NUTRIENTES } & DIETA 1 & DIETA 2 \\
\hline Humedad & 17,89 & 16,86 \\
\hline Proteína bruta & 18,50 & 24,69 \\
\hline Lípidos & 4,16 & 5,07 \\
\hline Fibra bruta & 4,74 & 2,68 \\
\hline Ceniza & 5,89 & 10,55 \\
\hline Carbohidrato & 48,82 & 40,15 \\
\hline Energía bruta $\mathrm{kcal} / \mathrm{g}$ & 345,95 & 353,78 \\
\hline
\end{tabular}

\section{RESULTADOS Y DISCUSIÓN}

En las figuras 1 y 2 se observan el crecimiento en peso de los peces. Estas figuras fueron diseñadas usando la siguiente ecuación:

1. $\mathrm{W}_{\mathrm{t}}=666.23\left[1-\mathrm{e}^{-0,1931(\mathrm{t}+11,5381)}\right]^{2,9405}$

2. $\mathrm{W}_{\mathrm{t}}=791.85\left[1-\mathrm{e}^{-0,0996(\mathrm{t}+2,8544)}\right]^{2,9144}$

El peso promedio inicial fue de 8,13 g para ambos experimentos (dieta 1 y dieta 2 ). Al final del estudio los pesos promedios fueron de 409,97 y 673,20 g, respectivamente (Tabla 3), y los pesos máximos asintóticos de 666,23 y 791,85 g (Tabla 4). 
Las figuras 1 y 2 , muestran que los peces crecieron en forma ascendente, siendo el experimento interrumpido cuando la producción máxima no se había alcanzado. Determinándose que el peso máximo asintótico (W¥) calculado de crecimiento fue bueno.

En la Tabla 4, se observa que los peces alimentados con la dieta 2 presentan mayores valores de longitud asintótica (L¥) que corresponden también a los mayores pesos asintóticos (W $¥$ ), lo cual no ocurre con los valores de $\mathrm{K}$, produciéndose en forma inversa, o sea, la dieta 2 con valores mayores en peso asintótico (W¥) tiene menores valores de $\mathrm{K}$ y viceversa.

Es importante determinar el crecimiento de los peces, porque nos permite verificar el efecto del alimento proporcionado, el cual está ligado con la densidad, Borges (1979).

En la Tabla 3 se observa que los peces alimentados con la dieta 2 tuvieron mejor crecimiento, comparados con los peces de la dieta 1, estadísticamente no hubo diferencia significativa $(\mathrm{P}>0,05)$ para los efectos de la proteína y de la energía.

Tabla 3. Promedios de longitud, peso y biomasa de Colossoma macropomum.

\begin{tabular}{|r|r|r|r|r|r|r|}
\hline & \multicolumn{3}{|c|}{ *DIETA1 } & \multicolumn{3}{c|}{ DIETA 2 } \\
\hline No. & $\mathbf{L}_{\mathbf{t}} \mathbf{c m}$ & $\mathbf{W}_{\mathbf{t}} \mathbf{g}$ & $\mathbf{B}_{\mathbf{t}} \mathbf{g}$ & $\mathbf{L}_{\mathbf{t}} \mathbf{c m}$ & $\mathbf{W}_{\mathbf{t}} \mathbf{g}$ & $\mathbf{B}_{\mathbf{t}} \mathbf{g}$ \\
\hline 0 & 7,81 & 8,13 & 23902 & 7,60 & 8,12 & 21463 \\
\hline 1 & 10,06 & 23,45 & 68943 & 11,78 & 34,06 & 90024 \\
\hline 2 & 13,50 & 54,85 & 161260 & 15,90 & 81,76 & 215846 \\
\hline 3 & 26,10 & 92,00 & 270000 & 18,36 & 122,50 & 323400 \\
\hline 4 & 17,63 & 107,80 & 316932 & 20,39 & 164,56 & 432438 \\
\hline 5 & 19,30 & 149,24 & 438000 & 23,59 & 234,90 & 620136 \\
\hline 6 & 20,66 & 176,88 & 520000 & 25,70 & 300,62 & 793630 \\
\hline 7 & 22,65 & 204,56 & 610406 & 27,46 & 355,86 & 939470 \\
\hline 8 & 22,74 & 246,00 & 723240 & 27,54 & 384,37 & 1014736 \\
\hline 9 & 24,57 & 258,00 & 758520 & 28,49 & 407,00 & 1074480 \\
\hline 10 & 25,00 & 281,00 & 826140 & 29,45 & 492,00 & 1298880 \\
\hline 11 & 25,11 & 304,40 & 894936 & 29,88 & 518,00 & 1367520 \\
\hline 12 & 27,98 & 409,97 & 1205312 & 33,17 & 673,20 & 1777288 \\
\hline
\end{tabular}

$* \mathrm{~L}_{\mathrm{t}} \mathrm{cm} .=$ Promedio de longitud total en centímetros

$\mathrm{W}_{\mathrm{t}} \mathrm{g}$. = Promedio de Peso Total en gramos

$\mathrm{B}_{\mathrm{t}} \mathrm{g} .=$ Promedio de Biomasa Total en gramos 
El análisis indica que la dieta 2, con el nivel de 24,69\% de proteína bruta, tuvo mejores efectos sobre el crecimiento de los peces que la dieta 1 , con 18,50\% de proteína bruta, y el nivel $353,78 \mathrm{kcal} / \mathrm{g}$ de energía bruta fue más eficiente que el de $345,91 \mathrm{kcal} / \mathrm{g}$.

La ganancia de peso/día de los peces de este experimento fue mayor, comparada con la de otros experimentos descritos por Saint-Paul \& Werder (1981), quienes indican ganancias de 0,2-0,9 g/día (Tabla 4).

La conversión alimenticia (Tabla 4) muestra que los peces que consumieron la dieta 2 fueron mejores que los de la dieta 1, (2,7 y 2,9), sin embargo, estos valores están dentro del rango satisfactorio. Las 2 dietas empleadas en estos experimentos dieron mejores índices de aprovechamiento, por los peces, que los citados por Da Silva et al.; (1974); Nuñez \& Salaya (1983); Saint-Paul \& Werder, 1981; Luna, 1987.

Tabla 4. Crecimiento de Colossoma macropomum .

\begin{tabular}{|l|l|l|}
\hline \multicolumn{1}{|c|}{ PARAMETROS } & \multicolumn{1}{|c|}{ DIETA 1 } & \multicolumn{1}{c|}{ DIETA 2 } \\
\hline Relación $\mathrm{L}_{\mathrm{t}}$ y Lt & $5,759+0,8262 \mathrm{~L}_{\mathrm{t}}$ & $3,6776+0,8960 \mathrm{~L}_{\mathrm{t}}$ \\
\hline Long. asint. máx. cm & 33,13 & 34,98 \\
\hline Relación $\mathrm{T}^{*}{\mathrm{y} \mathrm{L}_{\mathrm{t}}}^{\mathrm{K}}$ & $-0,2622-0,1931_{\mathrm{t}}$ & $-0,2843-0,0996$ \\
\hline $\mathrm{K}$ & $-0,1930507$ & $-0,0996108$ \\
\hline $\mathrm{Te}$ & 1,358056 & 2,8544184 \\
\hline $\mathrm{f}$ & 0,0225669 & 0,0250776 \\
\hline $\mathrm{q}$ & 2,9404615 & 2,9144355 \\
\hline Relac. Peso/Long. & $\mathrm{W}_{\mathrm{t}}=0,0226 \mathrm{~L}_{\mathrm{t}}^{2,9405}$ & $0,02508 \mathrm{~L}_{\mathrm{t}}^{2,9144}$ \\
\hline Peso asint. máx. g & 666,23 & 791,85 \\
\hline Conversión Aliment. & 2,9 & 2,7 \\
\hline Ganancia Peso/día g. & 1,1 & 1,8 \\
\hline
\end{tabular}

Los factores fisico químicos del agua, muestran el rango de variación del oxígeno disuelto del estanque de $2640 \mathrm{~m}^{2}$, que va desde 1,0 a 4,5 ppm, y del estanque de $2940 \mathrm{~m}^{2}$, que fluctúa de 1,5 a 1,9 ppm; valores aceptados por la especie estudiada, ya que en algunos casos ésta especie soporta valores menores de acuerdo a Braum \& Junk (1982), quienes encontraron valores de 0,5 ppm y los peces sobrevivieron (Tabla 5). 
Tabla 5. Promedios mensuales de los factores fisico-químicos de los estanques experimentales.

\begin{tabular}{|c|c|c|c|c|c|c|}
\hline \multirow[t]{2}{*}{ MESES } & \multicolumn{3}{|c|}{ ESTANQUE DE $2640 \mathrm{~m}^{2}$} & \multicolumn{3}{|c|}{ ESTANQUE DE $2940 \mathrm{~m}^{2}$} \\
\hline & Temp. ${ }^{\circ} \mathrm{C}$ & Cond. U/H & Oxig. ppm & Temp. ${ }^{\circ} \mathbf{C}$ & Cond.U/H & Oxig. ppm \\
\hline Abril 15 & 27,80 & 41,66 & 3,00 & 27,00 & 90,00 & 1,91 \\
\hline Abril 30 & 27,40 & 49,66 & 3,10 & 27,00 & 95,00 & 1,91 \\
\hline Mayo 15 & 26,00 & 30,66 & 2,00 & 26,00 & 85,00 & 1,90 \\
\hline Mayo 30 & 26,50 & 10,00 & 2,20 & 26,00 & 112,00 & 1,50 \\
\hline Junio 15 & 27,50 & 50,00 & 1,20 & 27,00 & 90,00 & 1,50 \\
\hline Junio 30 & 28,50 & 52,00 & 2,20 & 27,00 & 100,00 & 1,50 \\
\hline \begin{tabular}{|l|} 
Julio 15 \\
\end{tabular} & 28,50 & 19,50 & 4,20 & 28,50 & 28,00 & 3,50 \\
\hline Julio 30 & 27,00 & 40,25 & 2,00 & 28,00 & 42,00 & 2,50 \\
\hline Agosto 15 & 27,50 & 47,50 & 1,00 & 28,00 & 47,00 & 1,50 \\
\hline Agosto 30 & 27,50 & 25,00 & 3,50 & 28,00 & 50,00 & 1,50 \\
\hline Setiembre 15 & 27,00 & 26,33 & 3,10 & 26,50 & 55,00 & 1,50 \\
\hline Setiembre 30 & 27,66 & 28,00 & 3,40 & 26,50 & 55,00 & 1,50 \\
\hline
\end{tabular}

La temperatura osciló entre un promedio mínimo de $26,00^{\circ} \mathrm{C}( \pm 0,2)$ y un promedio maxímo de $28,50^{\circ} \mathrm{C}( \pm 3,1)$, considerándose temperatura normal para la especie estudiada. Los límites de la conductividad eléctrica del agua, en ambos experimentos, estuvieron dentro de los rangos aceptables. 
Figura 1. Crecimiento en peso de los peces con la dieta 1

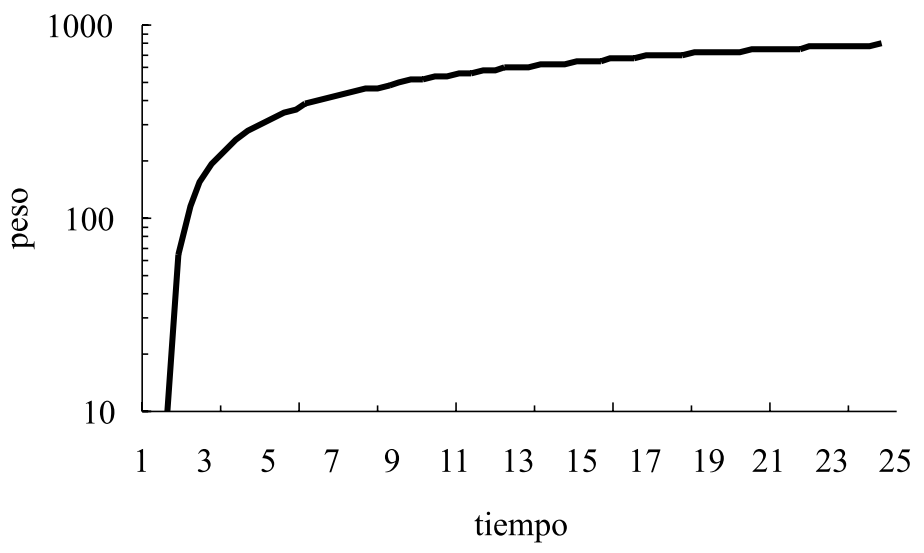

Figura 2. Crecimiento en peso de los peces con la dieta 2

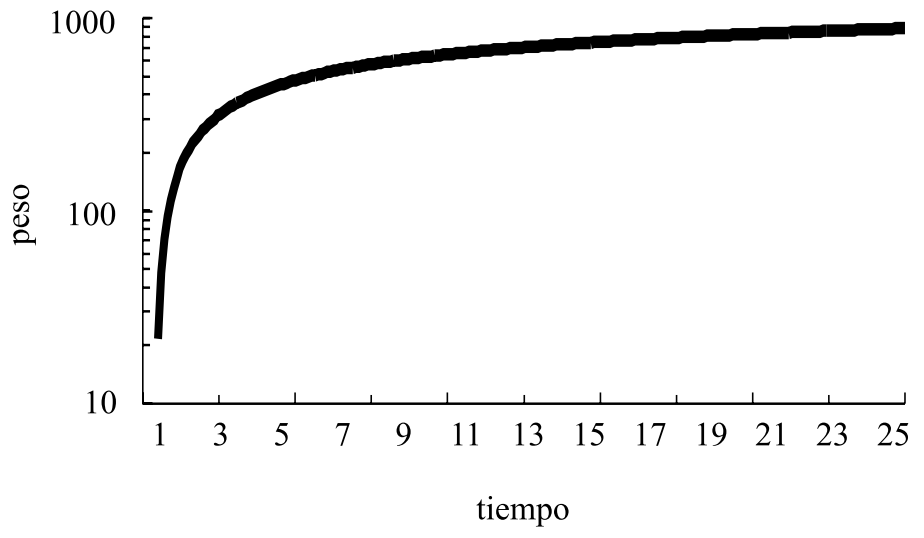




\section{BIBLIOGRAFÍA}

BRAUM, E. \& JUNK, W.J. 1982. Morphological Adaptation of two Amazonian Characoids (Pisces) For survivingin Oxygen Deficient Water. Internationale Rivue Der Gesamten Hidrobiologie. Vol. 67. No. 6:869-886.

BORGES, G.A. 1979. Aspectos quantitativos do cultivo de camarão Penaeus (=Melicertus) brasilensis (Latreille, 1817) em viveiro. Universidade Federal de São Carlos. Departamento de Ciências Biológicas. Dissertação de Mestrado. 63p.

DA SILVA, A.B. LOVSHIN, L.L. FERNÁNDEZ, J.A. \& CARNEIRO-SOBRINHO, A. 1974. Ensayo preliminar de cultivo en estanques de pirapitinga (Mylossoma bidens) y del tambaqui (Colossoma bidens) de la cuenca del río Amazonas. Actas del 2do. Simposio de Montevideo. FAO, Informes de Pesca No. 159, Vol. 1: 105-193.

LUNA, T. 1987. El efecto del contenido proteico y energético en alimentación artificial, sobre el crecimiento en Colossoma macropomun. Departamento de Piscicultura y Oceanografía. Universidad Nacional Agraria. La Molina. Lima. In: Proceeding of the Latin America Seminar of Aquacultura. 133136p.

MACEDO, E.M. 1979. Necessidades proteícas na nutrição de tambaqui Colossoma macropomun, Cuvier 1818, (Piscis, Characidae). Dissertação de Mestrado, FUCAV, UNESP/Jaboticabal, SP. 71pp.

MACEDO, E.M., CARNEIRO, D.J. \& CASTAGNOLLI, N. (1981) Necessidades proteícas na nutrição do tambaqui Colossoma macropomum, Cuvier 1818 (Piscis, Characidae). An. Simp. Bras. Aquic. II. Jaboticabal. SP. 77-78pp.

NÚÑEZ, J.M. \& SALAYA, J.J. 1983. Cultivo de cachama, (Colossoma macropomum, Cuvier 1818) en jaulas flotantes no rígidas en al represa de Guanapito-Estado de Guárico/Venezuela, V Simposium de la Asociación Latinoamericana de Acuicultura. Valdivia. Chile.

RANGEL, M.F.S.1987. Nutrição de peixes. In: Manual de pesca. Associação dos Engenheiros de Pesca. Fortaleza-CE. Ogawa \& Koike. Edits. pp.257-277. 
SAINT-PAUL, V. \& WERDER, V. 1977. Aspectos Generales sobre la piscicultura en Amazonas y resultados preliminares de experimentos de alimentación con raciones peletizadas con diferentes composiciones. Simp. Asoc. Lat. Acuic. I. Maracay-Venezuela, 22pp.

SAINT-PAUL, V. \& WERDER, V. 1981. The potential of some Amazonian fishies for warm water aquaculture. Proc. World Symp. On Aquaculture in heated Effluents and Recirculation Systems. Heenemenn \& CO, Berlin. Vol. II: 275-287pp

SALDAÑA, A.L. \& LÓPEZ, M.M.E. 1988. Formulación y evaluación de dietas para Colossoma macropomum, en México, An. VI Simp. Lat. e V Simp. Bras. de Aquic. Florianópolis. SC. 323-344 pp.

SANTOS, E.P. 1978. Dinámica de população aplicada à pesca e piscicultura. Hucitec, Ed. Universidade de São Paulo, 129 pp. 